\title{
Global Standards and Local Policies for School Diabetes Care
}

\author{
María J. Miranda Velasco', Maria Gloria Solís Galán², Enma Domínguez Martín³ \\ ${ }^{1}$ Department of Educational Sciences, University of Extremadura, Coordinator of Research Group Education \\ and Health Innovation, Cáceres, Spain \\ ${ }^{2}$ Department of Educational Sciences, University of Extremadura, Research Group Education and Health \\ Innovation, Cáceres, Spain \\ ${ }^{3}$ Research Group Education and Health Innovation, Cáceres, Spain \\ Email:mirandav@unex.es, glsolisg@unex.es, edominguezmartin86@gmail.com
}

Received *11 September 2015; accepted 8 December 2015; published 11 December 2015

Copyright (C) 2015 by authors and Scientific Research Publishing Inc.

This work is licensed under the Creative Commons Attribution International License (CC BY).

http://creativecommons.org/licenses/by/4.0/

(c) (i) Open Access

\begin{abstract}
Objectives: The purpose of this study is to analyse the practical implementation of regional and national policies through the Protocol of Care of Children and Adolescents in School (2010) in Extremadura Region (Spain), and to compare its contents with the international standards of diabetes care at school defined by American Diabetes Association and International Diabetes Federation. The measures not only affect the security and diabetes care, but also inclusion and the right to health. Methods: A documental comparative analysis between the local and international standards about diabetes care in school setting is carried out. This analysis is framed in a larger project focused on the study of health promoting school and diabetes education, in which perceptions of children and adolescents with diabetes, their parents and school staff were studied. Results: The Protocol of Care of Children and Adolescents in School (2010) contains some international recommendations about the care of T1DM at school, but in other cases the measures are non-specific. The distribution of responsibilities for care at school is unclear and no monitoring and evaluation indicators are defined. Some elements are identified to be implemented in the tool to favour the security, management of T1DM care and wellbeing. In general, these elements refer to school plan for diabetes care, school organization and teachers, and school community training. Conclusion: It is required to develop specific policies and decisive action to ensure the right to health of children with diabetes and the full application of international standards for diabetes care at school.
\end{abstract}

\section{Keywords}

Health Policy, Childhood and Adolescents, Diabetes Care, School Setting, Inclusion 


\section{Introduction}

The daily self-management of children and adolescents with T1DM is complex and variable [1] [2]. Besides the inherent requirement of the illness treatment in this period of life, the changes during the development of maturity require continuous adjustments in the treatment plan [3], and the aspects which affect the quality of childhood life need to be considered [4] [5].

Since children spend a significant part of the day in school [3], it is required to bring in their daily care measures into their school routine to accomplish an adequate metabolic control and the optimal development of treatment [6] [7]. At the same time, they should have the possibility of being fully and safely involved in all school activities [8]-[11].

The needs of children and adolescents with diabetes in school have been specifically identified in the main care standards [12]-[14]. These needs are based on the features of the treatment management (insulin therapy, blood glucose control, acute complications treatment and urgency situations and nutritional therapy) [6]-[10] [12].

Scientific literature, in line with current standards, underlines the importance of the training of the teachers and the educational school staff to acquire the basic knowledge about diabetes and the treatment of possible health emergencies [12] [14], to be able to guarantee the school work standardisation and the reduction of absenteeism related to diabetes [15]. Care of children with diabetes in school is an internationally shared concern, as it is shown in the continuous revisions of the care standards, guidelines and position statements from organisations like American Diabetes Association (ADA), American Association of Diabetes Educators (AADE), International Diabetes Federation (IDF) and International Society for Pediatric and Adolescent Diabetes (ISPAD), inter alia. However, there are very different legal and administration structures about diabetes care in school all around the world [16], despite having the American model as reference. Besides, the implementation in practice of recommendations is not being carried out in the desirable way as evidences the scientific literature reviewed [3] [5] [16]-[18]. Nowadays, the discrimination of children with diabetes in school continues to be a problem [8]-[10].

Despite the lack of comparative studies about the T1DM care policy and legal regulation in Europe, the international study "DAWN Youth" (2007-2009) reveals the existence of a great dispersion in terms of diabetes care policy and regulation measures in school in European countries studied [16].

In Spain, there are education policies that highlight the inclusion goals and the attention to student diversity [19] [20]. However, they only provide general prescriptive recommendations without specifying diabetic students' protection in school. Similarly, neither do they include evaluation indicators that guarantee the accomplishment of the objectives of care and educational inclusion.

In the health area, the National Diabetes Strategy (2012) recommends to promote the integration of children with diabetes through the design and development of a protocol of T1DM in school in all the Spanish Autonomous Communities.

The Community Autonomous of Extremadura has a Comprehensive Plan of Diabetes, which includes the national recommendations and proposes for the elaboration of the Protocol of Care of Children and Adolescents in School (2010) [21]. This Protocol is one of the few existing in Spain. It is the result of coordinated work held between the Regional Ministry of Education and the Regional Ministry of Health. The Protocol is proposed as a strategic solution to meet the care needs of the pediatric population with diabetes in the school setting. Its main objective is:

"To establish and promote specific measures of attention to children and adolescents with diabetes mellitus in the educational environment, and support for the entire educational community to promote their physical, social and emotional adjustment to illness as well as ensuring the control, security and equality opportunities of children and adolescents with DM in education" [21].

Currently, it has been implemented in 101 schools and involving a total of 171 children and adolescents with diabetes [22].

In our broader study about the needs and the quality of life of childhood and adolescence with diabetes [4], we wonder what the problem between educational policies and practices of care at school setting is.

The analysis and comparison of this regional document with the international care standards and recommendations are shown in the following paragraphs.

The objective of this study is to analyze the impact of international standards and recommendations of the di- 
abetes care at school in the regional health policy of Extremadura (Spain) through the measures defined in the instrument created for diabetes care at school called Protocol of Care of Children and Adolescents at School [21].

\section{Methods}

This is a descriptive study. Data collection was conducted through a documental analysis of the actual Spanish educational policy in relation to diabetes care at school. Specifically, the content of the Protocol of Care of Children and Adolescents at School (2010) [21], which has been developed by the Regional Ministry of Health of the Autonomous Community of Extremadura, was compared with the international standards and recommendations for diabetes care in childhood at school setting taken as reference in the document analyzed [9] [10] [23]. This study is part of a broader evaluation research related the diabetes education in children and adolescents carried out in Extremadura region, Spain (PRI09A156).

Specifically, it is discussed all necessary measures for diabetes care and safety, inclusion, family involvement, teachers training, school responsibilities, included in the tool in relation the international standards and recommendations of DM care in childhood and adolescence. The purpose is to support the decision-making process for the improvement of the effectiveness of the instruments created within the framework of the policies of Public Health administrations, at the service of the health and welfare of citizens from the international consensus and scientific evidences.The content analysis of the current legislation was made in 2013, after the implementation of the Protocol and the analysis of empirical study on which this paper is framed.

Analyzed dimensions and variables, according to the studied documents, are listed in Table 1 [9] [10] [23].

\section{Results}

Table 2 and Table 3 show the comparative analysis of the political document analysed between the Protocol of the Care of Children and Adolescents with diabetes in Schools of Extremadura Region [21] and the international standards by ADA and the IDF recommendations for diabetes care at school setting for Children and Adolescents. Implementation proposals are based in the family and the school responsibilities in the diabetes care [8]-[10] [23] [24].

Table 1. Analyzed dimensions and variables.

\begin{tabular}{|c|c|}
\hline Dimension: Family responsibilities & Dimension: School responsibilities \\
\hline Variables: Key aspects of diabetes care at school & Variables: Key aspects of diabetes care at school \\
\hline $\begin{array}{l}\text { 1. Participation in the development and implementation of personalized } \\
\text { School Care Plan }\end{array}$ & 1. Medical and educational records policy \\
\hline 2. Provision and use of diabetes care supplies & 2. Staff training in diabetes care \\
\hline 3. Maintenance of supplies & 3. Specification of minimum training content \\
\hline 4. Disposal of materials & 4. Medical treatment delivery and self-management policy \\
\hline 5. Health information registration & 5. Accommodation and privacy for self-management \\
\hline 6. Transmission of blood glucose values from school to parent/guardian & 6. Food school policy \\
\hline 7. Specification of necessary supplies & 7. Access to health services \\
\hline 8. Information delivery and training responsibilities & 8. Food classroom policy \\
\hline 9. Resources for action in medical emergencies & 9. Absence management policy \\
\hline 10. Food policy and family-school collaboration & 10. Free access to water and toilet \\
\hline \multirow[t]{3}{*}{ 11. Information policy and justification of absences for medical reasons } & 11. Medical supplies storage \\
\hline & 12. Food and nutritional information policy \\
\hline & $\begin{array}{l}\text { 13. Free access to medical supplies and participation in } \\
\text { school activities }\end{array}$ \\
\hline
\end{tabular}


Table 2. Family responsibilities in diabetes care at school.

\begin{tabular}{|c|c|c|c|}
\hline ADA 2004 & ADA 2008 & IDF 2005 & $\begin{array}{c}\text { Protocol for the Care of Adolescent } \\
\text { Children with Diabetes in School (2010) } \\
\text { Extremadura, (Spain) }\end{array}$ \\
\hline
\end{tabular}

1. Collaboration with the student's diabetes health care team to develop a personal Diabetes Medical Management Plan

2. All materials and equipment necessary for diabetes care tasks, including blood glucose testing, insulin administration (if needed), and urine or blood ketone testing

3. The parent/guardian is responsible for the maintenance of the blood glucose testing equipment.

4. Must provide materials necessary to ensure proper disposal of materials.

5. A separate logbook should be kept at school with the diabetes supplies for the staff or student to record test results.

1. Collaboration with the healthcare providers, school personnel, and the student for devised a

Diabetes Healthcare Plan

1.1. Transmitting the Diabetes Healthcare Plan and an emergency plan to the school

\section{Bringing diabetes supplies}

6. Blood glucose values should be transmitted to

the parent/guardian for review as often as requested.

7. Supplies to treat hypoglycaemia, including a

source of glucose and a glucagon emergency kit, if 7 .

indicated in the Diabetes Health Care Plan

8. Information about diabetes and the performance 8 . Transmitting diabetes information to of diabetes-related tasks the school

9. Emergency phone numbers for the

parent/guardian and the diabetes care team so that

the school can contact these individuals with

diabetes-related questions and/or

during emergencies

10. Information about the student's meal/snack schedule. The parent should work with the school to coordinate this schedule with that of the other students as closely as possible. For young children, instructions should be given for when food is provided during school parties and other activities.

11. In most locations and increasingly, a signed release of confidentiality from the legal guardian will be required so that the health care team can communicate with the school. Copies should be retained both at school and in the diabetes offices and health care professionals’ offices.
1. To inform the educational center about the diagnosis of the T1DM. Collaborate in the elaboration of a children and adolescents with diabetes personal plan (It is not specified who with, what or how)

2. Only after the needed care for the diabetes in school has been authorised by the School Board will all materials and essential equipment for the diabetes care be provided

2.1. Someone to go to administer insulin or other necessities when required by the school staff, and when the child/adolescent cannot do it by himself.

3. The parent is responsible for the maintenance of the blood glucose testing equipment.

4. The proper disposal of materials in a small container should be ensured working together with the reference nurse in DM.

5. To collaborate in the update of the student with DM's health card

6.-

8. Transmitting information about diabetes to school. Collaborating on everything required to control your son/daughter DM, with the teachers and the health professionals.

9.-

10. Collaborating on everything required to control your children's DM, with the teachers and the health professionals.

11. To inform teachers and school staff, at the discretion of the centre and under the family's authorization, about any student with DM.

11.1. To supply the student's medical and nurse reports relating to a student's illness, when they are required by the professionals who attend the child/adolescent.

11.2. To supply the teacher proof of student's absenteeism when it is due to the attendance of doctor or nurse's appointment or other reasons related to DM. 
Table 3. School responsibilities in the management of diabetes care.

\begin{tabular}{|lc}
\hline ADA 2004 & Protocol for the Care of Adolescent \\
Children with Diabetes in School \\
(2010) Extremadura, (Spain)
\end{tabular}

1.-----------------------------------

1. An individual Plan of diabetes care for the child or adolescent should be attached to the student academic record.

\section{2.}

2. Training to all adults who provide education/care for the student on the symptoms and treatment of hypoglycaemia and hyperglycemia and other emergency procedures.
2. Training of an adequate number of personnel
3. An adult and back-up adult(s) trained to 1)

perform fingerstick blood glucose monitoring and record the results; 2) take appropriate actions for blood glucose levels

outside of the target ranges as indicated in the student's

Diabetes Medical Management Plan; and 3) test the urine or

3

blood for ketones, when necessary, and respond to

the results of this test.

4. Immediate accessibility to the treatment of hypoglycaemia

by a knowledgeable adult

4.1. The student should remain supervised until appropriate treatment has been administered, and 4.2.

The treatment should be available as

close to where the student is as possible.

4.3. An adult and back-up adult(s) trained to administer glucagon, in accordance with the student's Diabetes Medical Management Plan.

4.4. If indicated by the child's developmental capabilities and the Diabetes Medical Management Plan, an adult and back-up adult(s) trained in insulin administration.

\section{A location in the school to provide privacy} during testing and insulin administration, if desired by the student and family, or permission for the student to check his or her blood glucose level and to take appropriate action to treat hypoglycaemia in the classroom or anywhere the student is in conjunction with a school activity, if indicated in the student's Diabetes Medical Management Plan.

6. An adult and back-up adult(s) responsible for the student who will know the schedule of the student's meals and snacks and work with the parent/guardian to coordinate this schedule with that of the other students as closely as possible. This individual also will notify the parent/ guardian ${ }^{6-}$ in advance of any expected changes in the school schedule that affect the student's meal times or exercise routine. Young children should be reminded of snack times.

7. Permission for the student to see school medical personnel, school nurse and other trained school personnel upon request.
4. Supervision or execution of diabetes tasks
2.1. It is only recommended an increase in the Regional Teaching training Plan, the number of educational activities in Health Education related to DM

(obesity, physical exercise promotion, healthy eating, diabetes education.

3.

4. To request emergency health care

4.1. To encourage the child/adolescent to be accompanied by a teacher or a social educator when he has to carry out a glycemic control, insulin administration or when a hypoglucemic situation is suspected.

4.2.

4.3.

4.4.

6--

7. Permission for the attendance to external medical appointments or other reasons related to DM

8. Permission, only when this is integrated in the Organisation and Operation Regulations in schools, to eat or to have a drink in class only to avoid a possible hypoglycaemia

It is considered bad monitoring when the frequency of this behaviour is high and the family must be reported. 


\section{Continued}

9. Permission to miss school without consequences for required medical appointments to monitor the student's diabetes management. This should be an excused absence with a doctor's note, if required by usual school policy.

10. Permission for the student to use the restroom and have access to fluids (i.e., water) as necessary.

11. An appropriate location for insulin and/or glucagon storage, if necessary.

12. Information on serving size and caloric, carbohydrate, and fat content of foods served in the school (27).

13. The student with diabetes should have immediate access to diabetes supplies at all times, with supervision as needed.

13.1. Provisions similar to those described

Above must be available for field trips, extracurricular activities, other school-sponsored events, and on transportation provided by the school or day care

facility to enable full participation in school activities.
9. Permission to allow the academic tests performance (exams, work handling, etc.) to be carried out later if the child/adolescent with DM condition requires and always when it is justified by document: hyperglycemia or hypoglycaemia, review medical appointments, etc.)

10. Only when these circumstances have been added to the Organisation and Operation Regulations in schools will permission be granted.

11. Only after getting from the School Board the permission for the admission of materials related to DM, will they count on a small refrigerator.

12. There is no specific information about the nutrients. It is only indicated that alternative elaboration menus should be ensured in the case that the student with DM uses the school cafeteria.

13. Only with the School Board permission can the admission of materials related to DM be authorised.

13.1. To encourage the child/adolescent to take part in all school activities, including days out and excursions, not specifying measures

The categories analysed in Table 2 refers to family/guardian responsibilities in the care of diabetes in school setting, Personal Diabetes Medical Management Plan, materials and equipment necessary for diabetes care tasks, information to the family, emergency phone numbers for the parent/guardian and the diabetes care team, and the emergency kit.

The categories analysed in Table 3 refers to the organizational school setting for provide the management of diabetes treatment, security, wellbeing; provider professional at school, educational responsibilities in the management of diabetes care, permissions for children at school, inclusion, and educational staff training.

\section{Discussion}

\subsection{The Regulation of Diabetes in the School Context}

The concern about the regulation of diabetes in school is a worldwide problem, where there is no unanimity in the management and each country has adopted different measures [16].

In the first DAWN Youth meeting in 2006, the educational centres were already identified as a priority objective for improvement; however, the current situation reveals that we are still at the same stage as we were years ago, as the evidence about the existence of structural, organisational, educational and attitudinal barriers are shown [16]

In general, in Spain, as it occurs in other countries, there are no state or community measures (administration or legal regulations) that guarantee the implementation and evaluation of the implementation of diabetes care in school.

\subsection{Protocol of Attention to Adolescent Children with Diabetes at School}

In Extremadura Region, the named Protocol of attention to adolescent children with diabetes in School [21] is considered the main reference for diabetes care in the school context. The results of the research carried out reveal that in most cases it is an unknown measure by the school personnel, and as it is designed, the safety and well-being of children with diabetes in school cannot be guaranteed despite being theoretically grounded in the 
standards of the ADA and the IDF.

The Protocol exposes that the approval of the fundamental measures related to diabetes treatment is due to the decision of the school itself. Therefore, the care and safety of children with diabetes are conditioned by the authorisation of the School Board in each centre, above their arising needs from the care and control of the illness. The decisions taken by this governing team consisting of representants of the educational and non-educational local community, affect the stocks and storage of the materials for the diabetes care in school, the administration of drugs, the meal intake in class and the reception of the specific refrigerator for the appropriate conservation of insulin and glucagon.

Another important gap in this Protocol is the lack in operability. The implementation is not monitored because there are no measures, nor indicators that guarantee it. On the other hand, the monitoring and the evaluation of it are totally unspecific.

In many cases establishing the roles of all involved does not identify the particular figure in charge of implementing actions or define the specific procedures to materialise them. The supporting functions of the diabetes care are defined ambiguously, not showing the differences between the responsibilities of the professionals and the education and health administration.

Training for diabetes care in school is neither guaranteed for teachers or non-teaching staff, it is only suggested as an increase to the general offers of training actions unspecified from the Teachers and Resources Centres. In practice, this fact is confirmed previous results from the largest study. Besides, recommending the cooperation of the associations of patients with DM and the associations of parents of students. Informative material is referred to as being be available to recipients; in any case this material is specific about T1DM in children and adolescents.

Versus the absence of a school nurse, the Protocol proposes the appointment of two people in charge of the care coordination in school: a nurse reference in diabetes in every health area and a reference person in diabetes in each school. The assumption of responsibility by this second figure, however, is voluntary. Previous training of both reference figures in the T1DM care is not established as a pre-requirement. Total or partial lack of a nurse in educational centres is a situation that affects most of the countries studied [6] [16] [17]. Therefore student attendance is devolved to teaching and administration staff [6], particularly to the family [25]. The role of a Credentialed Diabetes Educator in other countries like Australia and United States of America is considered as the ideal figure to provide the support required in the school environment [6].

Planning and implementation of specific measures like the Protocol is a step forward in the defense of the rights of children with diabetes, bur still shows important gaps. Regarding the fulfilment of the international care standards, to include control, evaluation and monitoring measures of treatment management in school to guarantee the safety and well-being of children with T1DM, besides ensuring a suitable academic performance [6] [8]-[10] [26]. Along the same line, other authors propose the implementation of auditing and/or feedback systems within an evaluation process orientated to improve quality [17].

Some contents of the Protocol seems more oriented as a means of protection against hypothetical legal responsibility of the school, instead of being a useful instrument to guarantee the safety, inclusion and sharing responsibilities. A contradiction is detected between child safety and inclusion objectives, care conditions at school, and the measures established.

The last International DAWN Youth study highlights the need to develop legislation measures to guarantee the distribution of responsibilities, the enforcement of care planning and the treatment management in the educational environment [16]. In another recent study carried out in the UK it is suggested that the policy and the legislative framework related to the diabetes management is not sufficient to ensure schools offer optimal care to children with diabetes [27]. The ambiguity of policies regarding the responsibilities in schools, leads to different care practice of diabetes in school [27].

\subsection{Practice and the Reality in Schools}

The models in the United States, Sweden, UK, the policies should make clear the role in school, identify the responsibilities for the care and the provision of medical care to support the optimal management of diabetes in schools [27]. Although, more investigation is required to provide more evidence about the reasons for the different diabetes treatment in different schools of the same country and in the world, the international policies of diabetes management in school should be developed to improve the consistency of care and ensure the equity for 
all children with diabetes [6].

It is recommended an interdisciplinary perspective and measures to guarantee the participation and collaboration between children, parents, educational staff and medical team to ensure the welfare [14], the integration of diabetes care in the school routine and establish a safe learning environment [6]-[11] [16].

\section{Relevance and Limitations of the Study}

The implications of the findings highlight the need to design systems for evaluating the practical implementation of policies related to health and educational care of children and adolescents with diabetes mellitus at school setting. The implementation measures must ensure the full inclusion of children with diabetes at school, welfare, security and optimal self-care and management of their treatment. In addition, the participation and coordination of all care settings are required.

These international care goals require continuous school staff training in health promoting school and specifically in diabetes care, a certain school organization based on a comprehensive model of education and new political commitments.

Limitations of the study are due to the analysis of the Protocol that has been focusing on specific documents relating to the care standards and guidelines of ADA and IDF used by the Protocol in their background and justification. Future editions of the Protocol must be analysed on the basis of their latest reviews. Also, the comparative analysis of the various documents existing in other regions or countries can provide a broader vision of care policy and legal regulation of diabetes care at school.

\section{Acknowledgements}

The Government of Extremadura and Funds FEDER (European Union) for funding the PRI09A156 Project: Virtual Platform to Support Diabetes Education in Childhood and Adolescence (PAED).

\section{References}

[1] Schwartz, F.L., Denham, S., Heh, V., Wapner, A. and Shubrook, J. (2010) Experiences of Children and Adolescents With Type 1 Diabetes in School: Survey of Children, Parents, and Schools. Diabetes Spectrum, 23, 47-55. http://dx.doi.org/10.2337/diaspect.23.1.47

[2] Faro, B., Ingersoll, G., Fiore, H. and Ippolito, K.S. (2005) Improving Students' Diabetes Management through School-Based Diabetes Care. Journal of Pediatric Health Care 19, 301-308. http://dx.doi.org/10.1016/j.pedhc.2005.03.004

[3] Tolbert, R. (2009) Managing Type 1 Diabetes at School: An Integrative Review. The Journal of School Nursing, 25, 55-61. http://dx.doi.org/10.1177/1059840508329295

[4] Miranda Velasco, M.J., Domínguez Martín, E., Arroyo Díez, F.J., Méndez Pérez, P. and González de Buitrago Amigo, J. (2012) [Health Related Quality of Life in Type 1 Diabetes Mellitus]. Anales de Pediatría, 77, 329-233.

[5] MacLeish, S.A., Cuttler, L. and Koontz, M.B. (2013) Adherence to Guidelines for Diabetes Care in School: Family and School Nurse Perspectives. Diabetes Care, 36, e52. http://dx.doi.org/10.2337/dc12-2083

[6] Marks, A., Wilson, V. and Crisp, J. (2013) The Management of Type 1 Diabetes in Primary School: Review of the Literature. Issues in Comprehensive Pediatric Nursing, 36, 98-119. http://dx.doi.org/10.3109/01460862.2013.782079

[7] American Association of Diabetes Educators (2008) Management of Children with Diabetes in the School Setting. AADE Position Statement. The Diabetes Educator, 34, 439-443. http://dx.doi.org/10.1177/0145721708317873

[8] American Diabetes Association (2014) Diabetes Care in the School and Day Care Setting. Diabetes Care, 37, S91-S96.

[9] American Diabetes Association (2008) Diabetes Care in the School and day Care Setting. Diabetes Care, 31, S79-S86.

[10] American Diabetes Association (2004) Diabetes Care in the School and Day Care Setting. Diabetes Care, 27, S122-S128.

[11] American Diabetes Association (2005) Care of Children and Adolescents with Type 1 Diabetes: A Statement of the American Diabetes Association. Diabetes Care, 28, 186-212. http://dx.doi.org/10.2337/diacare.28.1.186

[12] American Diabetes Association (2009) Standards of Medical Care in Diabetes_-2009. Diabetes Care, 32, S13-S61. http://dx.doi.org/10.2337/dc09-S013

[13] International Diabetes Federation (2011) Global IDF/ISPAD Guideline for Diabetes in Childhood and Adolescence. http://web.ispad.org/sites/default/files/idf-ispad_diabetes_in_childhood_and_adolescence_guidelines_2011.pdf 
[14] Pihoker, C., Forsander, G., Fantahun, B., Virmani, A., Luo, X., Hallman, M., Wolfsdorf, J. and Maahs, D.M. (2014) ISPAD Clinical Practice Consensus Guidelines 2014. The Delivery of Ambulatory Diabetes Care to Children and Adolescents with Diabetes. Pediatric Diabetes, 15, 86-101. http://dx.doi.org/10.1111/pedi.12181

[15] Gregg, E.W. and Albright, A.L. (2009) The Public Health Response to Diabetes-Two Steps Forward, One Step Back. JAMA, 301, 1596-1598. http://dx.doi.org/10.1001/jama.2009.519

[16] Lange, K., Jackson, C. and Deeb, L. (2009) Diabetes Care in Schools—The Disturbing Facts. Pediatric Diabetes, 10, 28-36. http://dx.doi.org/10.1111/j.1399-5448.2009.00613.x

[17] Edwards, D., Noyes, J., Lowes, L., Haf Spencer, L. and Gregory, J.W. (2014) An Ongoing Struggle: A Mixed-Method Systematic Review of Interventions, Barriers and Facilitators to Achieving Optimal Self-Care by Children and Young People with Type 1 Diabetes in Educational Settings. BMC Pediatrics, 14, 228. http://dx.doi.org/10.1186/1471-2431-14-228

[18] Lewis, D.W., Powers, P.A., Goodenough, M.F. and Poth, M.A. (2003) Inadequacy of In-School Support for Diabetic Children. Diabetes Technology \& Therapeutics, 5, 45-56. http://dx.doi.org/10.1089/152091503763816463

[19] Ley orgánica 2/2006, de 3 de mayo, de Educación. In: BOD, ed. num. 106, 04-May-2006.

[20] Ley 4/2011, de 7 de marzo, de educación de Extremadura. Comunidad Autónoma de Extremadura. In: DOE, ed. num. 47, 9-March-2011. https://www.boe.es/buscar/pdf/2011/BOE-A-2011-5297-consolidado.pdf

[21] Government of Extremadura (2010) Protocol of Care of Children and Adolescents in School (Protocolo de atención al niño/a y adolescente con diabetes en la escuela). Regional Ministry of Health and Dependency-Regional Ministry of Education, Government of Extremadura, Mérida, Extremadura.

[22] Government of Extremadura (2013) Extremadura Health Plan 2013-2020. Mérida, Extremadura.

[23] International Diabetes Federation (2005) The Rights of the Child with Diabetes in the School—Unsafe at School. Position Statement-Rights of Child with Diabetes in the School. http://www.unsafeatschool.ca/the-rights-of-the-child-with-diabetes-in-the-school

[24] American Diabetes Association (2013) Diabetes Care in the School and Day Care Setting. Diabetes Care, 36, S75-S79.

[25] Bodas, P., Marín, M. and Amillategui, B. (2008) Diabetes in the School. Perceptions of Children and Adolescents with Type 1 Diabetes. Avances en Diabetología, 24, 51-55.

[26] Dabelea, D., Mayer-Davis, E.J., Saydah, S., Imperatore, G., Linder, B., Divers, J., Bell, R., Badaru, A., Talton, J.W., Crume, T., Liese, A.D., Merchant, A.T., Lawrence, J.M., Reynolds, K., Dolan, L., Liu, L.L. and Hamman, R.F. (2014) Prevalence of Type 1 and Type 2 Diabetes among Children and Adolescents from 2001 to 2009. JAMA, 311, 17781786. http://dx.doi.org/10.1001/jama.2014.3201

[27] Marshall, M., Gidman, W. and Callery, P. (2013) Supporting the Care of Children with Diabetes in School: A Qualitative Study of Nurses in the UK. Diabetic Medicine, 30, 871-877. http://dx.doi.org/10.1111/dme.12154 